\title{
Personalidade e Comportamentos Problema: Um Estudo Comparativo com Adolescentes em Contexto Escolar
}

\author{
Personality and Problem Behavior: A Comparative Study with Adolescents \\ in School Context
}

\author{
Renato G. Carvalho* \& Rosa F. Novo \\ Centro de Investigação em Psicologia da Universidade de Lisboa, Lisboa, Distrito de Lisboa, Portugal
}

\begin{abstract}
Resumo
Neste estudo transversal analisámos a relação entre os comportamentos problema ao nível da indisciplina, desrespeito e conflituosidade dos adolescentes, em contexto escolar, e a personalidade e a psicopatologia, operacionalizadas nas dimensões PSY-5 do MMPI-A. Participaram no estudo 351 estudantes do $9^{\circ}$ ao $12^{\circ}$ ano, com idades compreendidas entre os 14 e os 18 anos, distribuídos em três grupos diferentes, de acordo com o reporte da frequência de comportamentos problema. Os instrumentos foram a versão experimental portuguesa do MMPI-A e um questionário sobre diferentes dimensões do percurso escolar, em que se incluem os comportamentos problema. Através de análise estatística multivariada, verificámos diferenças significativas entre os grupos nas dimensões PSY-5, num padrão em que o grupo de maior frequência de comportamentos problema apresenta resultados superiores no MMPI-A. Os resultados são interpretados numa perspetiva que sublinha a relevância da personalidade na adaptação à vida escolar, bem como a importância da existência de técnicas de avaliação psicológica de forma a detetar, em contexto de normalidade, estudantes em risco de desenvolvimento de trajetórias inadaptativas.

Palavras-chave: Personalidade, ajustamento escolar, externalização, MMPI-A.
\end{abstract}

\begin{abstract}
In this cross-sectional study we analyzed the relationship between problem behaviors in school context, such as indiscipline, disrespect or conflictuality, and personality and psychopathology, which were operationalized in the PSY-5 dimensions of the Minnesota Multiphasic Personality Inventory-Adolescent (MMPI-A). Participants were 351 students from $9^{\text {th }}$ to $12^{\text {th }}$ grades, aged from 14 to 18 years old, and distributed in three different groups, according to their report of problem behavior frequency. Instruments were the Portuguese experimental version of the MMPI-A and a questionnaire on several school dimensions, including problem behaviors. Multivariate statistical analysis of variance indicates significant differences between the groups in PSY-5 dimensions, in a pattern in which the group presenting the highest frequency of problem behaviors has higher results in the MMPI-A. Results are interpreted under a perspective that underlines the importance of personality in the comprehension of adaptation to school, as well as the relevance of psychological assessment techniques that make possible to detect, within normality contexts, students at risk of developing non-adaptive developmental pathways.

Keywords: Personality, school adjustment, externalization, MMPI-A.
\end{abstract}

Os padrões de comportamento, particularmente a existência de perturbações, correspondem a um marcador relevante da adaptação à escola e do sucesso académico, pelo que devem ser considerados quando analisamos o percurso escolar (Carvalho \& Novo, 2010). Os comportamentos problema dos estudantes podem expressar-se em diversas categorias, como o comportamento antissocial, problemas de conduta e perturbações de oposição ou

\footnotetext{
* Endereço para correspondência: Faculdade de Psicologia, Centro de Investigação em Psicologia, Universidade de Lisboa, Alameda da Universidade, Lisboa, Portugal 1649-013. E-mail: renatoggc@gmail.comerfnovo@ psicologia.ulisboa.pt.
}

indisciplina, os quais se incluem no domínio mais abrangente da externalização (Achenbach, 1991; Achenbach \& Edelbrock, 1978), isto é, do comportamento excessivo, problemático e de passagem ao ato.

Apesar de alguns comportamentos "difíceis", como o desrespeito pelos direitos dos outros ou pelas normas sociais, poderem em alguma medida ser considerados expectáveis e mesmo normativos na adolescência (Archer, 2005; Emond, Ormen, Veenstra, \& Oldehinkel, 2007), a sua frequência e intensidade merecem atenção, já que podem sinalizar perturbações significativas, com repercussões no desenvolvimento e no sucesso futuro. De facto, para além da associação entre os comportamentos problema e 
um conjunto de riscos de insucesso escolar, a investigação indica que estes problemas podem estender-se no tempo e ter impactos em diversos domínios da vida pessoal. Relativamente à extensão temporal, vários estudos reportam associações entre comportamentos problema em períodos de desenvolvimento e novos problemas no futuro (Bennett et al., 1999; Calkins \& Keane, 2009; Fergusson \& Woodward, 2000; Ruchkin, Koposov, Eisemann, \& Hägglöf, 2001), com alguns autores a mencionar a existência de uma estabilidade intraindividual nos padrões de comportamento ao longo do tempo (Woodward, Fergusson, \& Horwood, 2002). Quanto ao impacto dos comportamentos problema, muitos deles já na idade adulta, são alguns exemplos a maior probabilidade de problemas criminais, abuso de substâncias ou perturbações da personalidade (Barnown, Lucht, \& Freyberger, 2005). Neste contexto, importa compreender que fatores poderão estar associados à maior probabilidade de manifestação destes comportamentos problema e, particularmente, analisar a possibilidade de desenvolver metodologias que possam contribuir para a sua sinalização atempada.

\section{Comportamentos Problema e Características da Personalidade}

A literatura tem mostrado consistentemente que os problemas de externalização se relacionam com a personalidade em diferentes estádios de desenvolvimento. Vários estudos enfatizam como relevantes, para a compreensão de comportamentos problema nos indivíduos, os traços da personalidade, a predominância da emocionalidade negativa, a impulsividade e o sensation-seeking (Barnown et al., 2005; Cukrowicz, Taylor, Schatschneider, \& Iacono, 2006; Frick, 2004; Frick \& Dantagnan, 2005; Hill, 2002; Joyce \& Oakland, 2005; Miller \& Lynam, 2001; Ruchkin et al., 2001; Singh \& Waldman, 2010). A impulsividade refere-se a uma incapacidade de inibição e tendência para envolvimento imediato em situações, sem planeamento ou consideração por implicações potenciais desse envolvimento (Zuckerman \& Kuhlman, 2000), ao passo que o sensation seeking envolve a procura de sensações novas, intensas e variadas, suscetibilidade ao aborrecimento e disponibilidade para envolvimento em riscos pessoais, como consequência dessas experiências (Cazenave, Le Scanff, \& Woodman, 2007; Gute \& Eshbaugh, 2008; Rolison \& Scherman, 2002, 2003; Zuckerman \& Kuhlman, 2000).

Algumas destas características estão relacionadas com a preferência por atividades novas e perigosas, com a desinibição e ausência de sensibilidade à punição; todas elas são consistentes com um estilo designado frequentemente através de diferentes expressões - impulsivo, desinibido, temerário ou ainda desafiante (Frick, 2004; Frick \& Dantagnan, 2005) - e que tem sido relacionado com a expressão de problemas de comportamento.

Vários estudos longitudinais apoiam a ideia de que, desde logo na infância, a impulsividade é importante para o desenvolvimento de problemas de externalização (Barnown et al., 2005; Lynam \& Miller, 2004), sendo a baixa inibição comportamental vista como um antecedente potencial de problemas de conduta. Crianças e adolescentes impulsivos, especialmente quanto se associam a pares com problemas semelhantes, podem, por exemplo, estar em risco de rejeição por parte de outros colegas e de adultos, bem como de acentuação dos problemas comportamentais já existentes (Hill, 2002).

No âmbito dos modelos fatoriais da personalidade, os estudos têm vindo a identificar alguns traços que se associam negativamente aos problemas de comportamento, nomeadamente a Amabilidade e a Conscienciosidade, que se relacionam com o comportamento antissocial e com a agressividade (Miller, Lynam, \& Jones, 2008). Embora a Conscienciosidade nem sempre seja identificada como um preditor daqueles comportamentos, apesar de se correlacionar com eles, algumas das suas facetas, como a Deliberação apresentam já um valor preditivo. Tendo em conta que esta faceta envolve a "capacidade individual de pensar cuidadosamente antes de agir" (Costa \& McCrae, 1992 , p. 18), estes resultados contribuem para a consolidação da importância do controlo dos impulsos como uma característica significativa para a compreensão dos problemas de comportamento na adolescência.

É ainda de referir que têm sido relevados alguns traços, designadamente de insensibilidade e de ausência de culpa e de empatia, na distinção entre crianças e jovens com problemas de comportamento mais severos (Frick \& Dantagnan, 2005). Alguns destes estilos temperamentais distintos são mesmo consistentes com o construto de psicopatia (Frick, 2009; Gonçalves, 2007; Soeiro \& Gonçalves, 2010).

Dada esta associação entre características da personalidade e problemas de externalização, procurámos com este estudo, através do Minnesota Multiphasic Personality Inventory - Adolescent (MMPI-A; Butcher et al., 1992), cuja versão portuguesa está em processo de aferição (Silva, Novo, Prazeres, \& Pires, 2006), verificar se a frequência daqueles problemas se encontra relacionada com diferenças individuais ao nível da organização da personalidade. Neste sentido, consideramos possível, não só comparar os resultados da literatura internacional com os resultados obtidos com uma amostra em contexto português, como também analisar a eficácia da versão portuguesa do MMPI-A na diferenciação de indivíduos com diferentes padrões de comportamento, ainda que inseridos em contextos de normalidade e sem sinalização de psicopatologia. Este estudo pretendeu assim obter dados relevantes para a análise das características psicológicas dos adolescentes que apresentam e não apresentam problemas de comportamento, bem como evidências que apoiem a utilização do MMPI-A como uma prova compreensiva de avaliação psicológica, capaz de detetar diferenças, mesmo em contexto de normalidade e com uma população com a qual ainda não foi muito utilizado. 


\section{Método}

\section{Amostra}

A amostra foi constituída por 351 estudantes (212 do sexo feminino, o que corresponde a cerca de $60 \%$ ), a frequentarem o ensino básico ( $9^{\circ}$ ano; $\left.n=123\right)$, secundário $\left(10^{\circ}\right.$ ao $12^{\circ}$ ano; $\left.n=202\right)$ e profissional $(n=26)$. A maioria dos estudantes residia em áreas rurais $(n=223$, cerca de $64 \%$ ) e as suas idades encontram-se compreendidas entre os 14 e os 18 anos $\left(M_{\text {Idade }}=16\right.$ anos; $\left.D P=1,43\right)$. Verifica-se uma interação Idade x Nível de Escolaridade, $\chi_{(20, N=351)}^{2}=$ $223,27, p<0,001$, com um número superior ao esperado de estudantes com idade elevada para o nível de escolaridade em que se encontravam, o que está relacionado com o facto de cerca de metade da amostra apresentar pelo menos uma retenção no percurso escolar.

\section{Instrumentos}

Questionário sobre o Percurso Escolar (QPE; Carvalho \& Novo, 2010). Este questionário de autorrelato recolheu dados sobre diversas dimensões do percurso escolar, correspondendo uma delas à frequência de comportamentos problema (10 itens; $\alpha$ de Cronbach $=0,85$ ), que incluem comportamentos como agressões e brigas, indisciplina e falta de respeito em relação aos colegas e professores. Numa escala de tipo Likert, quatro alternativas de resposta eram possíveis, desde "Não Ocorreu" até "Ocorreu Frequentemente". A variável Comportamentos Problema correspondeu à média de todos aqueles itens, sendo que, quanto mais elevada a média, mais comportamentos problema foram reportados em contexto escolar. Foi a partir desta variável que se constituíram posteriormente três grupos de jovens (como referido na secção Procedimento e Análise de Dados), conforme a frequência de problemas identificados de problemas comportamentais.

Minnesota Multiphasic Personality Inventory Adolescent (MMPI-A; Butcher e colaboradores, 1992). De forma a avaliarmos as dimensões da personalidade e psicopatologia, utilizámos a versão portuguesa do MMPI-A (Silva e colaboradores, 2006), um inventário de autorrelato constituído por 478 itens. Neste estudo, recorremos às dimensões PSY-5 do MMPI-A (McNulty, Harkness, Ben-Porath, \& Williams, 1997), um modelo descritivo e dimensional de potenciais perturbações ao nível da personalidade, baseado num sistema concetual de cinco fatores principais (Archer, 2005) e que coloca a sua ênfase em traços da personalidade ou diferenças disposicionais específicas (McNulty e colaboradores, 1997). Descrevemos seguidamente estas cinco dimensões, bem como o seu número de itens e os alfas obtidos com esta amostra. A Agressividade (20 itens; $\alpha=0,729$ ) envolve a tendência para a hostilidade e comportamento combativo, com ênfase na agressividade instrumental e ofensiva, bem como sentimentos de grandiosidade face aos outros e desejo de poder e domínio social; o Psicoticismo (21 itens; $\alpha=0,728)$ associa-se a fenómenos sensoriais e percetivos pouco usuais, com suspeição em relação aos outros e probabilidade exibição de comportamentos de natureza psicótica, ansiedade e obsessões; a Desinibição (24 itens; $\alpha=0,721)$ é caracterizada por uma tendência para ações impulsivas, propensão para o risco, ausência de desejo por ordem e planos de ação e dificuldade no cumprimento de regras; o Neuroticismo (22 itens; $\alpha=0,739$ ) corresponde a uma disposição afetiva para experienciar emoções negativas, como ansiedade, nervosismo, preocupação e culpa o que leva a sofrimento interno; por fim, a Introversão (28 itens; $\alpha=0,735$ ) associa-se a uma reduzida emocionalidade positiva, à tendência global para não procurar ou gostar de experiências sociais (isolamento social e alienação) e ausência de energia para orientar-se para atividades e objetivos de vida.

\section{Procedimentos e Análise de Dados}

A aplicação dos questionários ocorreu em duas sessões e em contexto de turma, nos meses de maio e junho de 2010. O QPE foi aplicado na primeira sessão e o MMPI-A na segunda sessão. Após a recolha de dados, foi efetuada uma leitura ótica dos protocolos de MMPI-A, sendo os dados transferidos para uma base dados no SPSS, na qual foram também lançados os dados do QPE, depois de categorizados e cotados. Os protocolos de MMPI-A foram previamente avaliados relativamente aos indicadores de validade e de consistência de resposta.

O estudo, realizado com uma amostra transversal, é de natureza quantitativa e inscreve-se numa metodologia diferencial inter sujeitos, em que considerámos a análise de variância, especificamente a MANOVA, num desenho fatorial que tem como variáveis independentes os comportamentos problema e o sexo, e como variáveis dependentes as diferentes dimensões PSY-5. Efetuámos uma verificação preliminar das assunções da MANOVA, nomeadamente normalidade, linearidade, ausência de outliers univariados e multivariados, homogeneidade de matrizes de variância-covariância e ausência de multicolinariedade (Tabachnick \& Fidell, 2007).

Foi a partir da distribuição dos resultados da variável Comportamentos Problema que os jovens foram distribuídos por três grupos, de acordo com a frequência reportada de agressões e brigas, indisciplina e falta de respeito em relação aos colegas e professores: (a) o primeiro grupo (n =47), designado de "Frequência Muito Baixa ou Nula" correspondeu ao primeiro quartil; (b) o segundo grupo $(n=237)$, designado "Frequência Média", correspondeu ao segundo e ao terceiro quartis; (c) o último grupo $(n=$ 67), denominado "Frequência Elevada", correspondeu ao último quartil de distribuição dos resultados. A adoção desta estratégia de diferenciação dos jovens com base nos percentis deveu-se ao facto de os resultados médios serem baixos (i.e., em geral os jovens terem reportado uma baixa frequência de comportamentos problema), $M=1,5 ; D P=$ 0,45 , e de a distribuição dos resultados não ser normal, $D$ $=2,67, p<0,001$ (Kolmogorov-Smirnov). É ainda importante assinalar que, embora seja expectável a presença de quadros clínicos no terceiro grupo, este não corresponde 
Carvalho, R. G. \& Novo, R. F. (2014). Personalidade e Comportamentos Problema: Um Estudo Comparativo com Adolescentes em Contexto Escolar.

a um grupo com psicopatologia sinalizada nem diagnosticada, mas a jovens que reportaram maior frequência de problemas comportamentais em contexto escolar.

No estudo, partimos da hipótese global que as diferenças na personalidade se relacionam com os comportamentos problema, donde os adolescentes que reportam frequências mais elevadas destes problemas tendem a apresentar resultados mais elevados no MMPI-A. As nossas hipóteses específicas foram as seguintes: (a) Adolescentes com frequência elevada de comportamentos problema têm resultados significativamente superiores na Agressividade, sendo caracterizados por maior hostilidade, combatividade, desejo de domínio sobre os outros e disposição para experienciar sentimentos de zanga, quando comparados com os adolescentes sem comportamentos problema; (b) Adolescentes com frequência elevada de comportamentos problema têm resultados significativamente superiores na Desinibição, isto é, revelam maior impulsividade, dificuldade no cumprimento de regras e tolerância ao risco.

\section{Resultados}

Os resultados começam por mostrar a ausência de um efeito de interação Sexo x Comportamentos Problema, $F_{(10,682)}=1,20, p=0,282, \Lambda$ de Wilks $=0,965$, o que sugere a inexistência de efeitos de moderação do sexo nos resultados dos grupos de comportamentos problema no MMPI-A. Em relação aos comportamentos problema, verificam-se diferenças significativas entre os grupos nas variáveis dependentes, $F_{(10,682)}=6,81, p<0,001, \Lambda$ de Wilks $=0,827, \eta_{p}^{2}=0,09$, com uma dimensão do efeito que podemos classificar de moderada (Cohen, 1988).

Tabela 1

Diferenças entre os Grupos de Comportamentos Problema nas Dimensões da Personalidade PSY-5

\begin{tabular}{|c|c|c|c|c|}
\hline Dimensão & Grupo de Comp. Probl. ${ }^{\mathrm{a}}$ & $M(D P)$ & $F_{(2,345)}$ & $\eta_{p}^{2}$ \\
\hline \multirow[t]{3}{*}{ AGG. Agressividade } & (1) Muito Baixa/Nula & $6,50(2,94)$ & $17,36^{*}$ & 0,09 \\
\hline & (2) Média & $8,09(3,59)$ & & \\
\hline & (3) Elevada & $10,53(3,27)$ & & \\
\hline \multirow[t]{3}{*}{ PSYC. Psicoticismo } & (1) Muito Baixa/Nula & $4,55(3,13)$ & $9,71 *$ & 0,05 \\
\hline & (2) Média & $4,90(2,94)$ & & \\
\hline & (3) Elevada & $6,62(3,66)$ & & \\
\hline \multirow[t]{3}{*}{ DISC. Desinibição } & (1) Muito Baixa/Nula & $4,15(2,95)$ & $26,50 *$ & 0,13 \\
\hline & (2) Média & $5,80(3,00)$ & & \\
\hline & (3) Elevada & $8,92(3,56)$ & & \\
\hline \multirow[t]{3}{*}{ NEGE. Neuroticismo } & (1) Muito Baixa/Nula & $11,47(3,98)$ & 2,29 n.s. & 0,01 \\
\hline & (2) Média & $11,83(4,16)$ & & \\
\hline & (3) Elevada & $11,68(3,74)$ & & \\
\hline \multirow[t]{3}{*}{ INTR. Introversão } & (1) Muito Baixa/Nula & $7,57(4,97)$ & 1,13 n.s. & 0,00 \\
\hline & (2) Média & $6,42(3,76)$ & & \\
\hline & (3) Elevada & $6,39(3,67)$ & & \\
\hline
\end{tabular}

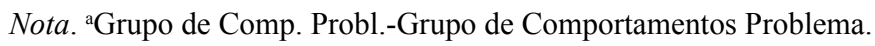

${ }^{*} p<0,001$.

As dimensões onde se observam diferenças significativas são a Desinibição, $F_{(2,345)}=26,50, p<0,001, \eta_{p}{ }^{2}=0.13$, a Agressividade, $F_{2,345)}=17,36, p<0,001, \eta_{p}^{2}=0,09$, e o Psicoticismo, $F_{(2,345)}=9,71, p<0,001, \eta_{p}{ }^{2}=0,05$. O efeito de maior magnitude é observado nos resultados da Desinibição, o que indica distinções entre os grupos de comportamentos problema ao nível da impulsividade, orientação para o planeamento e cumprimento de regras, propensão para o risco e moralidade prevalecente (moralidade mais ou menos convencional). Verificamos também diferenças significativas na hostilidade, combatividade e disposição para experienciar zanga, associados à Agressividade, bem como em características de alienação e desligamento da realidade, relacionados com o Psicoticismo. 
Numa análise post-hoc, através do teste de Tukey, verificamos que o terceiro grupo (frequência superior de comportamentos problema) se distingue de todos os restantes, com resultados mais elevados naquelas dimensões PSY-5 ( $p<0,001)$. No caso da Agressividade, verificamos ainda que o primeiro e o segundo grupos (respetivamente frequências baixa/nula e intermédia de comportamentos problema) se distinguem entre si $(p=0,012)$, o que ocorre também na Desinibição $(p=0,003)$. Em suma, a tendência global é para um aumento nos resultados médios nas dimensões PSY -5 em que foram encontradas diferenças significativas, à medida que transitamos do primeiro para o segundo grupo, e deste para o terceiro grupo de comportamentos problema. Estes resultados vêm ao encontro das nossas hipóteses, nomeadamente de que o grupo mais problemático, isto é, com frequência mais elevada de comportamentos problema ao nível da indisciplina, cumprimento de regras e do respeito pelos outros, apresenta resultados também mais elevados nas dimensões da personalidade. Para além do que hipotetizamos, os jovens com mais comportamentos problema têm ainda resultados mais elevados no Psicoticismo.

\section{Discussão}

Com este estudo procurámos analisar a relação entre a personalidade e os comportamentos problema em contexto escolar. Tivemos como principal objetivo verificar se a frequência elevada de problemas de externalização está relacionada com resultados distintos na personalidade, operacionalizada nas dimensões PSY-5 do MMPI-A. Como pudemos observar, verificou-se um padrão de diferenças significativas, o que sinaliza uma tendência para maiores dificuldades ao nível da organização da personalidade por parte de jovens com comportamentos problema frequentes. Apesar de, como referimos anteriormente, esta tratar-se de uma amostra sem sinalização de psicopatologia, pudemos verificar diferenças significativas nos resultados, sendo que, quanto mais problemático o grupo, mais elevada foi a média nas dimensões PSY-5. A nosso ver, esta circunstância ilustra a eficácia da prova na deteção de diferenças interindividuais, ainda que situadas num quadro global de população sem referenciação clínica.

De entre as dimensões da personalidade, foi na Desinibição e na Agressividade em que os resultados mais se destacaram, o que globalmente reflete distinções importantes entre os jovens ao nível do controlo dos impulsos, sensation seeking e baixa aversão ao risco, bem como hostilidade, combatividade e propensão para problemas interpessoais (Archer, 2005). No caso da Desinibição, a magnitude do efeito foi moderada, refletindo a importância desta dimensão para a manifestação de comportamentos problema no percurso escolar. Verificámos ainda uma associação positiva entre o Psicoticismo e os comportamentos problema, o que ilustra que os jovens com estes problemas têm maior tendência para apresentarem características de alienação social e da realidade, bem como bizarria no com- portamento e suspeição em relação aos outros. Considerando a literatura, estes resultados apoiam os estudos que têm mostrado a associação entre problemas comportamentais, por um lado, e características como a impulsividade, desinibição, menor conscienciosidade e menor amabilidade, por outro (Barnown et al., 2005; Cukrowicz et al., 2006; Frick, 2004; Frick \& Dantagnan, 2005; Hill, 2002; Joyce \& Oakland, 2005; Latzam, Vaidya, Clark, \& Watson, 2011; Lynam \& Miller, 2004; Miller \& Lynam, 2001; Nelson, Stage, Duppong-Hurley, Synhorst, \& Epstein, 2007; Ruchkin et al., 2001; Singh \& Waldman, 2010). Além disso, estes resultados obtidos com uma amostra de jovens sem sinalização psicopatológica vêm reiterar a importância que a investigação tem atribuído à personalidade na explicação e compreensão das alterações comportamentais (e da probabilidade da sua ocorrência) na adolescência (Gute \& Eshbaugh, 2008; Hill, 2002; Joyce \& Oakland, 2005; Miller \& Lynam, 2001; Ruchkin et al., 2001). Com efeito, os resultados que obtivemos evidenciam que a forma como a personalidade se está a organizar tem correlatos ao nível da adaptação dos adolescentes aos diferentes contextos de desenvolvimento, isto é, a personalidade contribui para o modo como decorrem as transacções entre os jovens e os seus contextos de vida.

É, por isso que, neste contexto, que salientamos a importância da avaliação de jovens com sintomatologia significativa e com características potencialmente de risco para o desenvolvimento daquelas alterações comportamentais, como sejam aquelas associadas aos resultados mais elevados nas dimensões da personalidade e da psicopatologia. A atenção a estes jovens e a preocupação com a sua avaliação justifica-se pela necessidade de deteção precoce, de prevenção e intervenção posterior, no sentido de evitar trajetórias inadaptativas (Frick, 2004; Frick \& Viding, 2009) ou cascatas desenvolvimentistas em que se acumulem e potenciem dificuldades ao longo do tempo. Até porque os padrões sintomáticos de alterações comportamentais podem apresentar evoluções negativas para situações que comprometam de forma irreversível a saúde pessoal ou ainda para perturbações de natureza antissocial e/ou de mesmo psicopática, que têm o seu início em períodos anteriores e muito precoces do desenvolvimento (Frick, 2009; Gacono \& Hughes, 2004; Kotler \& McMahon, 2005).

A importância de se identificarem estes casos de forma atempada reside ainda no facto de jovens com padrões comportamentais problemáticos estarem inseridos em contextos onde muitas patologias passam despercebidas e onde as intervenções, por se dirigirem à normalidade, não mostram surtir efeito (Gacono \& Hughes, 2004). Apesar de, neste estudo, o grupo mais problemático não corresponder necessariamente a um grupo clínico, os dados mostram uma tendência clara para resultados mais elevados nas dimensões da psicopatologia. Temos, por isso, uma circunstância em que jovens inseridos numa amostra normal e em contextos de normalidade (escolar) produzem resultados que se encaminham num sentido da 
Carvalho, R. G. \& Novo, R. F. (2014). Personalidade e Comportamentos Problema: Um Estudo Comparativo com Adolescentes em Contexto Escolar.

sinalização de sintomatologia clinicamente significativa. Neste contexto, o MMPI-A foi eficaz na distinção destas dificuldades e padrões de comportamento, o que oferece indicadores de utilidade deste instrumento para a prática e para a intervenção - sobretudo quando, em comparação aos adultos, é frequentemente mais difícil discriminar a normalidade e as alterações à mesma no comportamento dos adolescentes (Archer, 1984, 1997).

Por fim, este estudo não deixa de apresentar algumas limitações, que deverão ser tidas em consideração. Sublinhamos que os dados foram recolhidos exclusivamente através de instrumentos de autorrelato, pelo que a nosso ver será útil realizar futuramente mais investigações com recurso a outras fontes de informação, por exemplo, reportes de pais, professores e pares, e eventualmente com recurso à combinação de métodos qualitativos e quantitativos, os quais nos permitiram captar uma maior diversidade $\mathrm{e}$ especificidade de padrões de comportamento, numa perspetiva idiográfica. Para além disso, tendo em conta que a amostra utilizada neste estudo é transversal, consideramos que será ainda importante realizar estudos longitudinais que, num enfoque da psicopatologia do desenvolvimento, nos permitam compreender a diversidade de trajetórias de desenvolvimento dos jovens e a influência dos problemas de comportamento nas mesmas.

\section{Referências}

Achenbach, T. M. (1991). Manual for the Child Behavior Checklist/4-18 and 1991 Profile. Burlington, VT: University of Vermont.

Achenbach, T. M., \& Edelbrock, C. S. (1978). The classification of child psychopathology: A review and analysis of empirical efforts. Psychological Bulletin, 85, 1275-1301.

Archer, R. P. (1984). Use of the MMPI with adolescents: A review of salient issues. Clinical Psychology Review, 4 241-251.

Archer, R. P. (1997). Future directions for the MMPI-A: Research and clinical issues. Journal of Personality Assessment, 68, 95-109.

Archer, R. P. (2005). MMPI-A: Assessing adolescent psychopathology ( $3^{\text {rd }}$ ed.). Mahwah, NJ: Lawrence Erlbaum.

Archer, R. P., Gordon, R. A., Giannetti, R. A., \& Singles, J. M. (1988). MMPI scales clinical correlates for adolescent inpatients. Journal of Personality Assessment, 53, 707-721.

Barnown, S., Lucht, M., \& Freyberger, H. (2005). Correlates of aggressive and delinquent conduct problems in adolescence. Aggressive Behavior, 31, 24-39.

Bennett, K. J., Lipman, E. L., Brown, S., Racine, Y., Boyle, M. H., \& Offord, D. R. (1999). Predicting conduct problems: Can high-risk children be identified in kindergarten and grade 1? Journal of Consulting and Clinical Psychology, 67(4), 470-480.

Butcher, J. N., Williams, C. L., Graham, J. R., Archer, R. P., Tellegen, A., Ben-Porath, Y. S., \& Kaemmer, B. (1992). Minnesota Multiphasic Personality Inventory - A (MMPIA): Manual for administration, scoring, and interpretation. Minneapolis, MN: University of Minnesota Press.
Calkins, S. D., \& Keane, S. P. (2009). Developmental origins of antisocial behavior. Development and Psychopathology, 21(4), 1095-1109.

Carvalho, R. G., \& Novo, R. F. (2010, dez.). Personalidade e percurso académico: Estudos com adolescentes portugueses. Trabalho apresentado no I Simpósio de Investigação em Psicologia da Universidade de Lisboa, Lisboa, Portugal.

Cazenave, N., Le Scanff, C., \& Woodman, T. (2007). Psychological profiles and emotional regulation characteristics of women engaged in risk-taking sports. Anxiety, Stress, \& Coping, 20(4), 421-435.

Cohen, J. (1988). Statistical power analysis for the behavioral sciences. Hillsdale, NJ: Erlbaum.

Costa, P. T., Jr., \& McCrae, R. R. (1992). Revised NEO personality inventory (NEO-PI-R) and NEO Five-Factor inventory (NEO-FFI): Professional Manual. Odessa, FL: Odessa Psychological Assessment Resources.

Cukrowicz, K. C., Taylor, J., Schatschneider, C., \& Iacono, W. G. (2006). Personality differences in children and adolescents with attention-deficit/hyperactivity disorder, conduct disorder, and controls. Journal of Child Psychology and Psychiatry, 47(2), 151-159.

Emond, A., Ormen, J., Veenstra, R., \& Oldehinkel, A. J. (2007). Preschool behavioral and social-cognitive problems as predictors of (pre)adolescent disruptive behavior. Child Psychiatry and Human Development, 38, 221-236.

Fergusson, D. M., \& Woodward, L. J. (2000). Educational, psychosocial, and sexual outcomes of girls with conduct problems in early adolescence. Journal of Child Psychology and Psychiatry, 41(6), 779-792.

Frick, P. (2004). Developmental pathways to conduct disorder: Implications for serving youth who show severe aggressive and antisocial behavior. Psychology in the Schools, 41(8), 823-834.

Frick, P. (2009). Extending the construct of psychopathy to youth: Implications for understanding, diagnosing, and treating antisocial children and adolescents. The Canadian Journal of Psychiatry, 54(12), 803-812.

Frick, P. J., \& Dantagnan, A. L. (2005). Predicting the stability of conduct problems in children with and without callousunemotional traits. Journal of Child and Family Studies, 14(4), 469-485.

Frick, P. J., \& Viding, E. M. (2009). Antisocial behavior from a developmental psychopathology perspective. Development and Psychopathology, 21(4), 1111-1131.

Gacono, C. B., \& Hughes, T. L. (2004). Differentiating emotional disturbance from social maladjustment: Assessing psychopathy in aggressive youth. Psychology in the Schools, 41(8), 849-860.

Gonçalves, R. A. (2007). Promover a mudança em personalidades anti-sociais: Punir, tratar e controlar. Análise Psicológica, 4(25), 571-583.

Gute, G., \& Eshbaugh, E. M. (2008). Personality as a predictor of hooking up among college students. Journal of Community Health Nursing, 25, 26-43.

Hill, J. (2002). Biological, psychological and social processes in the conduct disorders. Journal of Child Psychology and Psychiatry, 43(1), 133-164.

Joyce, D., \& Oakland, T. (2005). Temperament differences among children with conduct disorder and oppositional defiant disorder. The California School Psychologist, 10, 125-136. 
Kotler, J. S., \& McMahon, R. J. (2005). Child psychopathy: Theories, measurement, and relations with the development and persistence of conduct problems. Clinical Child and Family Psychology Review, 8(4), 291-325.

Latzam, R. D., Vaidya, J. G., Clarck, L. A., \& Watson, D. (2011). Components of disinhibition (vs. Constraint) differentially predict aggression and alcohol use. European Journal of Personality, 25, 477-486.

Lynam, D. R., \& Miller, J. D. (2004). Personality pathways to impulsive behavior and their relations to deviance: Results from three samples. Journal of Quantitative Criminology, 20(4), 319-341.

McNulty, J. L., Harkness, A. R., Ben-Porath, Y. S., \& Williams, C. L. (1997). Assessing the personality psychopathology five (PSY-5) in adolescents: New MMPI-A scales. Psychological Assessment, 9(3), 250-259.

Miller, J. D., \& Lynam, D. (2001). Structural models of personality and their relation to antisocial behavior: A meta-analytic review. Criminology, 39(4), 765-798.

Miller, J. D., Lynam, D. R., \& Jones, S. (2008). Externalizing behavior through the lens of the Five-Factor Model: A focus on Agreeableness and Conscientiousness. Journal of Personality Assessment, 90(2), 158-164.

Nelson, J. R., Stage, S., Duppong-Hurley, K., Synhorst, L., \& Epstein, M. H. (2007). Risk factors predictive of the problem behavior of children at risk for emotional and behavioral disorders. Exceptional Children, 73(3), 367-379.

Rolison, M. R., \& Scherman, A. (2002). Factors influencing adolescent decisions to engage in risk-taking behavior. Adolescence, 37(147), 585-596.

Rolison, M. R., \& Scherman, A. (2003). College student risk takin from three perspectives. Adolescence, 38(152), 689-704.

Ruchkin, V. V., Koposov, R. A., Eisemann, M., \& Hägglöf, B. (2001). Conduct problems in Russian adolescents: The role of personality and parental rearing. European Child and Adolescent Psychiatry, 10, 19-27.

Silva, D., Novo, R., Prazeres, N., \& Pires, R. (2006). Inventário Multifásico da Personalidade-Adolescente: Versão Experimental Portuguesa. Lisboa, Portugal: Centro de Investigação em Psicologia, Universidade de Lisboa.

Singh, A. L., \&Waldman, I. D. (2010). The etiology of associations between negative emotionality and childhood externalizing disorders. Journal of Abnormal Psychology, 119(2), 376-388.

Soeiro, C., \& Gonçalves, R. A. (2010). Estado da arte do conceito de psicopatia. Análise Psicológica, 1(28), 227-240.

Tabachnick, B., \& Fidell, L. S. (2007). Using multivariate statistics $\left(5^{\text {th }}\right.$ ed.). New York: Pearson.

Woodward, L. J., Fergusson, D. M., \& Horwood, L. J. (2002). Deviant partner involvement and offending risk in early adulthood. Journal of Child Psychology and Psychiatry, 43(2), 177-190.

Zuckerman, M., \& Kuhlman, D. M. (2000). Personality and risktaking: Common biosocial factors. Journal of Personality, 68(6), 999-1029. 